Results 180 patients participated in the study: 95 patients were male and 85 were female. The combined mean age was 38.9 years (range of 15-85 years). 112 patients had IBD and 68 had viral hepatitis. The mean age for the IBD patients was 39 years (range 16-85 years) and the mean age for the hepatitis patients was 38.6 years (range 15-63 years). 46 (43.75\%) of the IBD patients and 49 (72\%) of the hepatitis patients were male.

In our study, $82.1 \%$ of the IBD and $72.1 \%$ of the viral hepatitis patients used one or more social networking site. Facebook was the most popular site. $29.5 \%$ of the IBD and $33.8 \%$ of the viral hepatitis patients used social media for support with their illness. $80.4 \%$ of the IBD patients and $72.9 \%$ of the viral hepatitis patients said they would be happy with some form of social media interaction by healthcare professionals. $84.8 \%$ of the IBD patients and $72.1 \%$ of the viral hepatitis patients were in favour of a specific social media website for their disease.

Conclusion A large proportion of patients with IBD and viral hepatitis already use social networking sites. This study suggests that the majority of both IBD and viral hepatitis patients would welcome the use of social media as part of their illness management. There are already some social media sites that have been setup for these patient groups. Increasing the awareness of these sites and further research investigating the integration of social media into the current management of both these patient groups is needed.

Disclosure of Interest None Declared.

\section{PTH-074 OF WHAT VALUE IS A 1:1 MULTIDISCIPLINARY WEIGHT MANAGEMENT CLINIC?}

doi:10.1136/gutjnl-2013-304907.561

1."D Turner, ${ }^{2} \mathrm{~S}$ Jones, ${ }^{2} \mathrm{~S}$ Syal, ${ }^{2} \mathrm{~J}$ Gray, ${ }^{2} \mathrm{~J}$ Reynolds, ${ }^{2} \mathrm{~A}$ Weaver, ${ }^{2} \mathrm{~N}$ Haboubi. ${ }^{1} \mathrm{Cardiff}$ Medical School, Cardiff; ${ }^{2}$ Aneurin Bevan Weight Management Clinic, Ebbw Vale, UK

Introduction Obesity management in Wales includes provision of a 1:1 Multidisciplinary Weight Management Clinic (MDWMC). Strategic management of obesity in Wales is guided by The All Wales Obesity Pathway and recommends MDWMCs for people with obesity who have one or more co-morbidities and who have tried several interventions without success, or who have complex emotional relationships with food. ${ }^{1}$ This service evaluation aimed to assess physiological benefits associated with attendance at a MDWMC. It also aimed to collect qualitative data in an attempt to explain any reasons for achieving benefits.

Methods An approved questionnaire was used to conduct semistructured interviews with 180 patients attending the MDWMC at Aneurin Bevan Hospital, Ebbw Vale, Wales. Quantitative data were tabulated and a thematic analysis was performed on free-text responses to collate qualitative data.

Results The MDWMC supports weight loss with $95 \%$ of patients reporting loss. For those whom baseline data was available 73\% lost at least $5 \%$ of initial body weight. $88 \%$ of patients prefer individual appointments and over $90 \%$ of patients who see each team member find consultations useful. $69 \%$ of patients report improved health mainly due to a decrease in obesity-related symptoms, and of patients taking obesity-related medication $48 \%$ report a reduction in dose of medication for asthma, $42 \%$ report a reduction in dose of antidepressants and $36 \%$ report a reduction in dose for medication for diabetes. Of employed patients $30 \%$ report a reduction of days taken off work due to sickness. $96 \%$ of patients would recommend the clinic to others. Conclusion A 1:1 Multi-disciplinary Weight Management Clinic provides value in reducing obesity and symptoms of obesity-related diseases. It also is a treatment choice favoured by patients.

Disclosure of Interest None Declared.

\section{REFERENCE}

Welsh Assembly Government. All Wales Obesity Pathway. 2009. Accessed at: http://wales.gov.uk/topics/health/improvement/index/pathway/?lang = en

\section{PTH-075 YOU'VE GUT MAIL: A GASTROENTEROLOGY EMAIL HELPLINE IS EFFICIENT AND COST-EFFECTIVE IN RESOLVING PATIENT QUERIES AND REDUCING NON- ELECTIVE INPATIENT BED DAYS}

doi:10.1136/gutjnl-2013-304907.562

1."J L S Ooi, 'M Vinayaga-Pavan, 'D C K Koh, 'V Morgan, 'V S Wong. 'Gastroenterology, Whittington Health NHS Trust, London, UK

Introduction Electronic patient-provider communication may be a convenient, cost-effective complement to standard healthcare services $^{1}$. We piloted a dedicated gastroenterology (GI) email helpline to increase accessibility for patients and medical colleagues seeking specialist advice.

Methods Retrospective study of activity records of our GI specialist nurse-led email helpline across 40 months (Jan 2008-Jul 2010; Feb-Dec 2012). Data for 2012 was analysed to demonstrate efficiency. From our highest frequency user group - inflammatory bowel disease (IBD) patients - we identified a cohort of 21 users that had contact with our department 6 months before their index email, comparing hospital utilisation rates then and in the 6 months afterward (post-intervention). Analysis was by Mann-Whitney and $\chi^{2}$ tests. Cost savings were estimated based on Department of Health Reference Costs 2011/12.

Results The helpline received 264 emails from 153 users over 40 months: $73 \%$ from patients, $20 \%$ from GPs, $7 \%$ from others. Of 126 patients, $57 \%$ were female, $43 \%$ male. Mean age was 42.4 years (range 18-82 years). 2012 data analysis: Of 72 email queries, $72 \%$ were successfully resolved electronically. Mean turnaround time was 2.2 working days (range 0-9). 69\% (50 emails) concerned general advice, medications or results, queries that are conventionally handled in outpatients (OP; £141/appointment) or in telephone clinic (TC; $£ 55 /$ appointment). Only $8.5 \%$ of email queries subsequently required TC encounters; another $10 \%$ proceeded to OP. By approximating 1 hour's work per week for a GI specialist nurse at $£ 22 /$ hour $^{2}$, we estimate the email service cost $£ 1144$ in 2012 , plus $£ 1317$ for ensuing TCs and OPs, a total of £2461. This compares favourably to $£ 2750$ to answer the 50 queries by TC alone, or $£ 7050$ by $\mathrm{OP}$ alone. Highest uptake was among the IBD subgroup: 49 users generated 129 emails over 40 months. In the 6 months pre- \& postindex email, our identified cohort $(n=21)$ had similar rates of clinic attendance (41 vs 53 appts, $p>0.05$ ), DNAs ( 3 vs 3 ) and A\&E attendance ( 4 vs 0 visits, $p>0.05$ ). Reduction in non-elective inpatient bed days was significant (34 vs 4 days, $p<0.0001$; $£ 271 /$ day), representing savings of $£ 8130$ over 6 months.

\section{Reasons behind Email Queries}

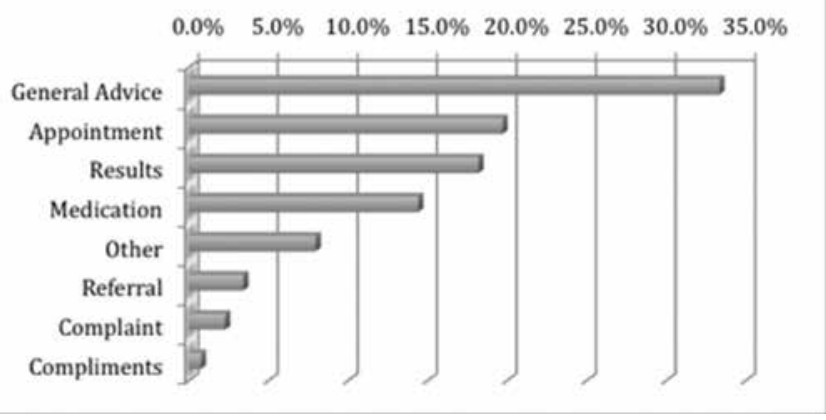

\section{Abstract PTH-075 Figure 1}

Conclusion Our GI email helpline has proven to be popular and economical. Most queries were resolved electronically, significantly reducing unscheduled inpatient bed days. We are planning a user 
satisfaction survey to measure quality. A prospective study is warranted as our service expands.

Disclosure of Interest None Declared.

\section{REFERENCES}

1. Impact of electronic messaging on the patient-physician interaction. Wallweiner $M$ et al. J Telemed Telecare. 2009; 15(5):243-250

2. Royal College of Nursing (2012) Inflammatory bowel disease nursing, London: RCN. Publication code 004197

\section{PTH-076 UNIVERSITY HOSPITAL SOUTHAMPTON IBD PORTAL PILOT- AN INNOVATIVE IT TOOL TO PROMOTE PATIENT SELF-CARE}

doi:10.1136/gutjnl-2013-304907.563

1." M Naghibi, ${ }^{2} \mathrm{M}$ Bettey, ${ }^{2} \mathrm{~F}$ Catherine, ${ }^{2} \mathrm{~J}$ Swabe, ${ }^{2} \mathrm{~J}$ Hunter, ${ }^{2} \mathrm{~K}$ Hamer, ${ }^{2} \mathrm{~F} \mathrm{~J}$ Cummings. 'Gastroenterology; ${ }^{2}$ University Hospital Southampton, Southampton, UK

Introduction Chronic disease management represents a big challenge to the NHS. The IBD standards specify the use of IT to support patient care and to optimise clinical management through data collection and audit. The development of innovative patient care pathways are required to meet these challenges as well as the Quality, Innovation, Productivity and Prevention (OIPP) agenda. 'My Health Record' is a secure web-based service built on the Microsoft HealthVault platform, which allows storage of health information from many sources in one secure online location. The IBD team and UHS IT department, in collaboration with web developers, GetReal, have designed this pilot website with the objective of improving patient access and care. Methods The IBD portal aims to provide an email based 'Flareline', record current and past medication history, inform patients of upcoming outpatient and endoscopy appointments, allow patient access to verified relevant investigation results, food/stool/health diaries and to provide tailored care plans with email and SMS reminders. We are taking advantage of existing innovative technologies, such as Smartphones capable of 'Near Field Communication' (NFC) and NFC enabled weight scales. These weight scales upload data directly to patient records, where aims and parameters are set, which alert the clinician to the progress of the patient.

Results The pilot IBD portal was launched in September 2012. We have recruited $\mathrm{n}=55$ patients over 5 months, with $\mathrm{n}=19$ patients completing the registration process. The most commonly used function of the service to date has been the email 'Flareline' and messaging service. These enquiries were answered with in one day for 'Flareline' messages and 1.8 days for non-urgent messages. Three patients have been supplied with NFC enabled weight scales with all patients using the devices regularly. The data collected using the NFC devices has lead to reliable clinical data and timely changes in treatment, particularly dietetic input.

Conclusion IBD is a chronic disease with a spectrum of clinical activity effecting quality of life and occurs in a significant proportion of patients in working age. The development of a web-based IBD portal is an innovative addition to IBD services with a potential to improve patient care and will lead to the development of new patient care pathways in collaboration with local care commissioning groups. We aim to improve cost effectiveness by reducing outpatient visits, reducing work load from phone based flareline enquiries and, provide more information on local IBD services for patients. Challenges to the IBD pilot so far have been to engage patients in this new model of care for chronic disease management.

Disclosure of Interest None Declared.

\section{PTH-077 THE CHANGING FACE OF CLOSTRIDIUM DIFFICILE INFECTION}

doi:10.1136/gutjnl-2013-304907.564

1."N M Joshi, 'J Seale, ${ }^{2}$ S Das. ' Centre for Digestive Diseases, Barts and The London School of Medicine and Dentistry; ${ }^{2}$ Department of Medical Microbiology, Barts Health NHS trust, London, UK
Introduction C.difficile infection (CDI) is the most common identified cause of antibiotic associated diarrhoea and carries a significant mortality. Several reports have demonstrated that exogenous infection plays an important role in the spread of CDI. Reports show that ribotype 027 has been responsible for large outbreaks of CDI and is associated with a poorer outcome.

Methods All cases of CDI over a 9 month period (ending August 2012) were cultured and typed by the London reference laboratory. For each case, retrospective data on patient demographics, admission dates, ward and clinical team were analysed.

Results 32 new cases of CDI occurred of which 22(69\%) could be ribotyped. All cases had had antibiotic exposure. Average age: 67 years, $27 \%$ of cases were from patients admitted to critical care and $13 \%$ were under elderly care. $27 \%$ of all cases were community and $73 \%$ hospital acquired. 12 ribotypes were seen (table 1), 1 case of type 027 . There were no cases of CDI of the same ribotype originating in the same clinical area or under the care of the same clinical team within 30 days of each other. 1 patient (ribotype 015) underwent colectomy for colonic perforation secondary to extensive pseudomembranous colitis with co-existing diverticular disease. There was $16 \%$ overall mortality on index admission with 1 death indirectly attributable to CDI (ribotype 020).

Abstract PTH-077 Table 1 No predominating ribotype was seen, 1 case of type 027

\begin{tabular}{lcccccccc}
\hline Ribotype & 002 & $\mathbf{0 0 3}$ & $\mathbf{0 1 4}$ & $\mathbf{0 1 5}$ & $\mathbf{0 2 0}$ & $\mathbf{0 2 7}$ & $\mathbf{1 0 6}$ & $\mathbf{0 2 3 , 0 3 1 , 0 5 6 , 1 7 6 , 4 1 1}$ \\
\hline No of cases & 4 & 2 & 3 & 2 & 3 & 1 & 2 & 1 case of each \\
\hline
\end{tabular}

Conclusion In contrast to previous literature, type 027 was not the predominant ribotype seen in our cohort. The case requiring colectomy was type 015 and the death indirectly attributed to CDI was also not caused by type 027 . This demonstrates a possible shift in the epidemiology of CDI. The groups most at risk were patients admitted to critical care and those under the care of the elderly care physicians with an overall $16 \%$ mortality whilst still admitted. There was little evidence of cross-infection and most cases were endogenously acquired indicating that infection prevention and control methods being practised at our Trust are effective. These findings also suggest that the main cause of CDI in this study arises from selection pressure secondary to antimicrobial use and emphasises the importance of antibiotic stewardship in the prevention and control of this infection.

Disclosure of Interest None Declared.

\section{Inflammatory bowel disease}

\section{PTH-078 A PROSPECTIVE EVALUATION OF THE PREDICTIVE VALUE OF FAECAL CALPROTECTIN IN QUIESCENT CROHN'S DISEASE}

doi:10.1136/gutjnl-2013-304907.565

1,"L A Smith, 'G Naismith, ${ }^{2} \mathrm{~S}$ Barry, ${ }^{1} \mathrm{~J}$ I Munro, 'S Laird, ${ }^{3} \mathrm{~K}$ Rankin, ${ }^{\prime} \mathrm{J}$ Morris, ${ }^{4} \mathrm{~J}$ W Winter, 'D R Gaya. ' Gastroenterology, Glasgow Royal Infimary; ${ }^{2}$ Robertson Centre for Biostatistics, University of Glasgow; ${ }^{3}$ Clinical Biochemistry, Glasgow Royal Infimary; ${ }^{4}$ Gastroenterology, University of Glasgow, Glasgow, UK

Introduction Studies have suggested that faecal calprotectin (FC) levels may increase early in inflammatory bowel disease relapse before the patient is symptomatic and thus may be useful to identify patients at a higher risk of relapsing. The purpose of this study was to evaluate the role of FC in predicting relapse in patients followed up for a minimum of 12 months and to ascertain the best cut-off for this in our cohort of adult patients with quiescent Crohn's disease (CD).

Methods Patients with CD in clinical remission were recruited and followed up prospectively for a minimum of 12 months. Participants 